\title{
GROWTH OF INFANT FED STARTER FORMULA CONTAINING MILK FAT GLOBULE MEMBRANES
}

\author{
C. Billeaud ${ }^{1}$, G. Puccio $^{2}$, E. Saliba ${ }^{3}$, B. Guillois ${ }^{4}$, S. Pecquet ${ }^{5}$, P. Steenhout ${ }^{5}$ \\ ${ }^{1}$ Hopital des Enfants, CHU Pellegrin, Bordeaux, France, ${ }^{2}$ Departimento Materno Infantile, Universita Degli \\ Studi di Palermo, Palermo, Italy, ${ }^{3}$ Hopital Clocheville, CHU de Tours, Tours, ${ }^{4}$ Hopital Clemenceau, CHU \\ de Caen, Caen, France, ${ }^{5}$ Nestec Ltd, Vevey, Switzerland
}

Objective: To assess growth of infants fed starter formula supplemented with milk fat globule membranes (MFGM).

Methods: Healthy, full term newborns ( $\mathrm{n}=199$, ITT population) were enrolled the $14^{\text {th }}$ day of their child's life and randomly assigned between 3 exclusively formula fed groups: one control group (commercial starter IF) and 2 experimental groups i.e. starter IF supplemented with 2 different MFGM fractions. Growth was assessed by body weight gain, length and head circumference measurements during the 4-month intervention period. Incidence of morbidity was appraised.

Results: After 4-month intervention treatment, no significant effect in infant body weight gain was observed when comparing each experimental groups versus the control and all z-scores both for male and females stayed between the interval of $+/-1$ during the whole interventional period. In addition, no statistical difference was observed between groups on the other anthropometric measurements (head circumference and length) during the intervention period.In summary, non inferiority in growth was established between each experimental groups versus the control at the end of the 4 months intervention period (Non-inferiority margin was pre-defined as $\Delta=-3 \mathrm{~g} /$ day). On the other hand, no statistically significant difference of adverse events was detected between all three groups.

Conclusion: Growth of newborns receiving IF containing MFGM was non inferior to the growth observed in controls and within the limits defined by WHO growth reference curves. 